\title{
The effect of laser acupuncture on hypoxia tolerance and inflammation reaction in mice with optical fiber acupuncture needle intra body
}

\author{
Shupeng $\mathrm{Liu}^{* * *}$, , Minmin $\mathrm{Xu}^{*}$, Xiumei $\mathrm{Cao}^{\dagger}$, Junyi Hou ${ }^{\dagger}$, Na Chen*, ${ }^{*}, \|$, Fufei Pang*, \\ Zhenyi Chen*, Xiangfei Yang*, Tingyun Wang* and Jianshe Yan ${ }^{\dagger, \boldsymbol{\Phi}, \|}$ \\ *Key Laboratory of Specialty Fiber Optics \\ and Optical Access Networks \\ School of Communication and Information \\ Engineering, Shanghai University \\ 333 Nanchen Road, Shanghai 200444, P. R. China \\ ${ }^{\dagger}$ Shanghai Institute of Immunology \\ Department of Immunology and Microbiology \\ Shanghai Jiao Tong University School of Medicine \\ 280 South Chongqing Road, Shanghai 200025, P. R. China \\ †iusp@shu.edu.cn \\ \$na.chen@shu.edu.cn \\ Tyanjianshe@sjtu.edu.cn
}

Received 7 January 2016

Accepted 4 April 2016

Published 22 April 2016

\begin{abstract}
Hypoxia is a disorder in which the tissues are not oxygenated adequately. The lack of oxygen may cause irreversible damage to the major organs such as the brain, heart and lungs. In severe cases, coma, seizures, and death may happen. In addition to the routine medication, acupuncture treatment has been applied as a complementary treatment to counter hypoxia. In this paper, the optical fiber acupuncture needle was fabricated using the optical fiber imbedding into the metal capillary tube with needle tip, therefore made it easy to insert into the body for the laser treatment. The laser optical fiber needle treatment to the ST36 acupoint conducted with the laser irradiation intra body. The normobaric hypoxia tolerance test results in mice show that the optical fiber needle treatment to the ST36 acupoint with laser acupuncture appears to improve the tolerance to hypoxia. The mice treated with laser acupuncture expressed high level of IL- $1 \beta$ in serum. Our results suggest that laser optical fiber needle acupuncture may serve as a potential treatment for hypoxia.
\end{abstract}

Keywords: Laser acupuncture; optical fiber; acupuncture needle; hypoxia; inflammation.

${ }_{\text {Corresponding authors. }}$

This is an Open Access article published by World Scientific Publishing Company. It is distributed under the terms of the Creative Commons Attribution 4.0 (CC-BY) License. Further distribution of this work is permitted, provided the original work is properly cited. 


\section{Introduction}

Acupuncture treatment has been well established as an effective therapy method by the clinic applications in China for thousands of years. Acupuncture is a fast, convenient and economical treatment method with numerous effective clinical validations. $^{1,2}$ Recently, acupuncture has been broadly used in clinical treatment abroad including America. ${ }^{3}$ Furthermore, laser acupuncture has been developed to stimulate acupuncture points on the outer skin without infection or pain, ${ }^{4}$ and used to improve the treatment of brain function, ${ }^{5-7}$ dental disease,,${ }^{8,9}$ and obesity. ${ }^{10}$ The laser stimulation is an alternative method for children with needle phobia. The combination of laser stimulation of CV 24 and acupressure of PC 6 is more effective than the laser stimulation of CV 24 alone. ${ }^{8}$ Interestingly, it was found that the laser stimulation of acupoint (LR14, CV14, LR8 and HT7) activates the frontal-limbic-striatal brain regions, with the pattern of neural activity somewhat different for each acupuncture point. ${ }^{11}$ The laser acupuncture has been clinically applied particularly in primary care since the 1970s. ${ }^{12}$ Over the years, many studies have been reported about the therapy effects and the mechanism of the laser acupuncture. ${ }^{13-18}$ However, because the depth of body that laser can reach is usually limited by the obstruction of skin, the effectiveness of the laser acupuncture treatment is reduced, especially if the locations of acupoints are deep in the body.

We speculate that the optical fiber needle treatment with delivering laser light directly to the acupoint intra body, combining the skills of traditional acupuncture, may lead to an improved therapy of the laser acupuncture. In this work, we set up the laser acupuncture needles with optical fiber that facilitates the laser to penetrate deeply into the body, and evaluate its therapy by acupuncture of ST36 in mice. We show that mice treated with laser acupuncture appear more tolerant to hypoxia and have high level of IL-1 $\beta$, a master cytokine involved in inflammation, in the serum. Our data suggest that the optical fiber needle treatment to the ST36 acupoint with laser acupuncture could improve the tolerance to hypoxia for mice, and the system may provide potential strategies for the treatment of hypoxia.

\section{Materials and Methods \\ 2.1. Fabrication of the laser acupuncture needles}

The stainless steel tube was selected with the diameters of $0.31 \mathrm{~mm}$ (inner) and $0.5 \mathrm{~mm}$ (outer). It was stretched, pulled and heated at the same time with a small flame injector of the self-designed device. After grinding the needle tip with machine to remove the oxide layer on the surface of the acupuncture needle, the peeled optical fiber was inserted into the hollow pipe needle. Then, the tail of acupuncture needle was sealed using strong adhesive glue to bond the fiber and the acupuncture needle.

The acupuncture needle device was made of the light source $(633 \mathrm{~nm}$ laser) connected with the acupuncture needle though the multimode fiber pigtail, as shown in Fig. 1. The light output power is $10 \mathrm{~mW}$ with the spot diameter of $0.01 \mathrm{~cm}$, and the power density is $31.85 \mathrm{~W} / \mathrm{cm}^{2}$.

\subsection{Animal experiments}

All mouse experiments were in compliance with ethical regulations and were approved by the Institutional Animal Care and Use Committee (IACUC) of Shanghai University. Twelve-week-old male C57BL/ 6 mice were purchased from Shanghai Silaike experimental animal Co. Ltd China. The animals were fed as experimental animals and treated according to the accepted international

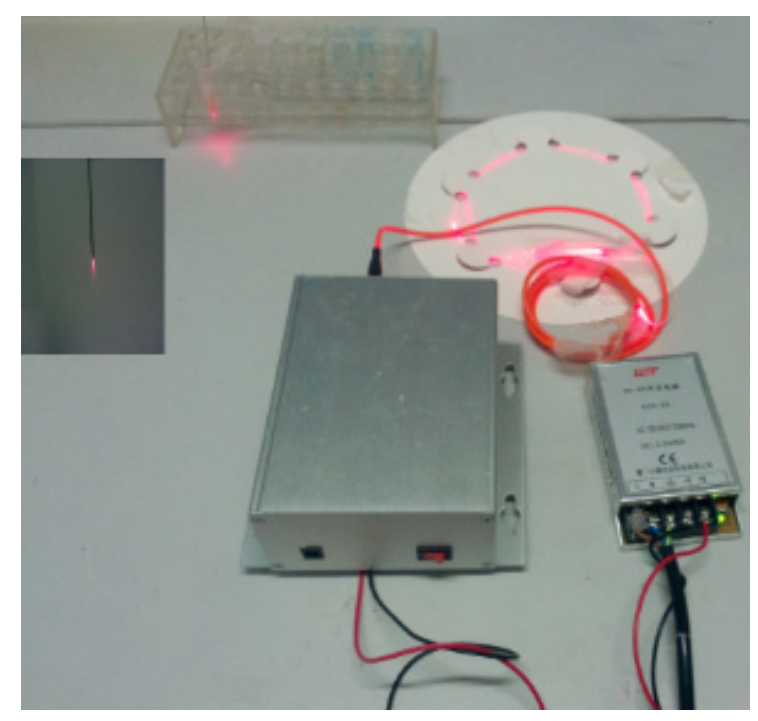

Fig. 1. Image of the acupuncture needle device. 
criteria. Animals were subjected to a $12: 12 \mathrm{~h}$ lightdark cycle in a temperature-controlled $\left(23^{\circ} \mathrm{C}\right)$, pathogen-free animal facility at Shanghai University. In total 18 healthy (SPF) C57BL/6 mice (12 weeks old, body weight $26.5 \pm 2.5 \mathrm{~g}$ ) were recruited in this study. The mice were divided into three groups: the control group (Control, $n=6$ ), the hollow acupuncture group (Hollow, $n=6$ ), and the laser optical fiber acupuncture group (Laser, $n=6$ ).

The acupuncture points selected were the position of the mouse ST36, which is $2 \mathrm{~mm}$ lateral to the anterior tubercle of tibia. ${ }^{10}$ The ST36 of each leg was treated on alternate days. As the left ST36 is selected on the first day of treatment, the right ST36 will be selected on the second day, then the left on the third day, and so on. Before treatment, Hollow group and Laser group on local skin of acupoint area underwent shaving and disinfection. The laser wavelength selected was $633 \mathrm{~nm} \mathrm{He}-\mathrm{Ne}$ laser and the needle sterilized with sterilization alcohol. Before acupuncture, the mice were injected with $0.1 \mathrm{ml} 7 \%$ chloral hydrate anesthesia water solution. The mice was shaved around the acupuncture points, and sterilized with $75 \%$ alcohol cotton at the acupoint.

The specific experimental operation process of each group is as follows (see Fig. 2.):

Control group A: the mice were fixed in the fixator, and then the mice were fixed with adhesive tape for $10 \mathrm{~min}$, once a day, a total of 10 times.
Hollow group B: mice were fixed in mice in the fixator, and then the mouse hind leg is fixed by adhesive tape, using sterile cotton swab to disinfect around ST36. Then, the disinfection hollow needle was bilaterally inserted at ST36, retaining the needle for $10 \mathrm{~min}$, once a day, for 10 times in total.

Laser group C: the mice were fixed in mice in the fixator, and then the mouse hind leg is fixed by adhesive tape, using sterile cotton swab to disinfect around ST36. Then, connected to the disinfection laser fiber needle device, and then stabbed into the mice's ST36, retaining the needle for $10 \mathrm{~min}$, once a day, for 10 times in total. The laser irradiation wavelength is $633 \mathrm{~nm}$. The output power of the laser is $10 \mathrm{~mW}$, the irradiation time is $10 \mathrm{~min}$, and the power density is about $31.85 \mathrm{~W} / \mathrm{cm}^{2}$.

\subsection{ECG detection}

Three days after the acupuncture finished, the heart rate was detected in mice. The mice were anesthetized with $0.01 \mathrm{~mL} / \mathrm{g}$ by intraperitoneal injection of $3 \%$ chloral hydrate, then the mice were fixed and ECG recorded (see Fig. 3).

\subsection{Normobaric hypoxia tolerance test}

Jars with volume of $250 \mathrm{ml}$ were selected for hypoxia tolerance test for mice, and Vaseline and seals were used to improve the sealing effect. Before sealing the jars, the equivalent soda lime wrapped

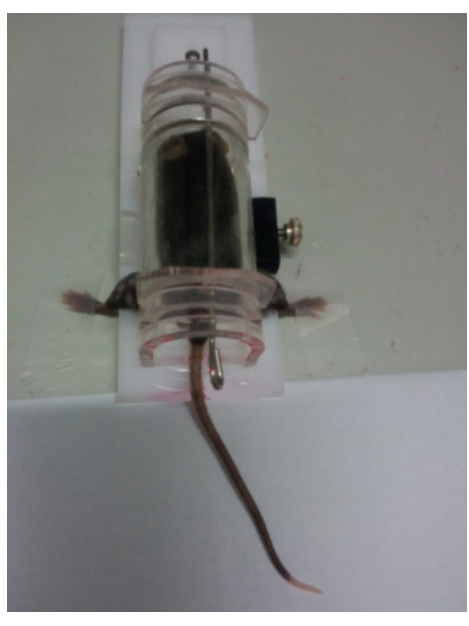

(a)

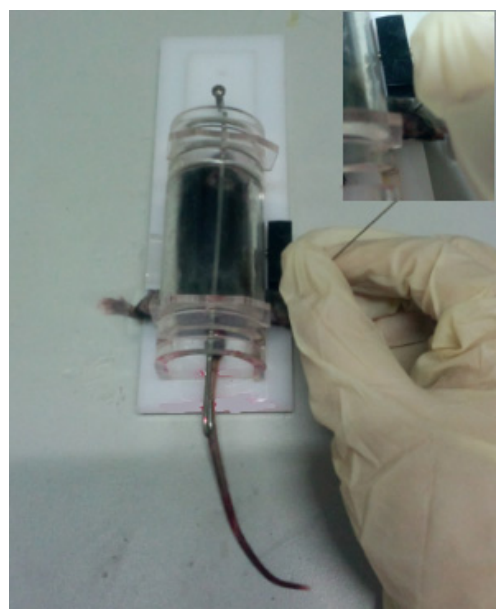

(b)

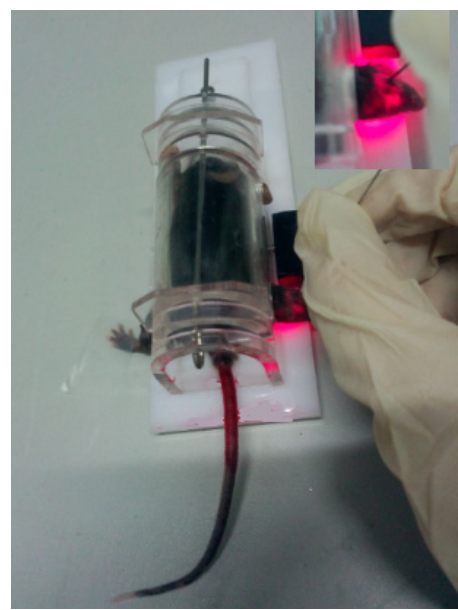

(c)

Fig. 2. The specific experimental operation process of each group. (a) Control group: the mice were fixed in the fixator, and then the mice were fixed with adhesive tape for $10 \mathrm{~min}$; (b) Hollow group: After mice were fixed, then applied the hollow acupuncture needling around ST36 for 10 min. (c) Laser group: Fixed mouse, then, connected the disinfection laser fiber needle device, and then stabbed into the mice's ST36, retaining the needle for $10 \mathrm{~min}$. 


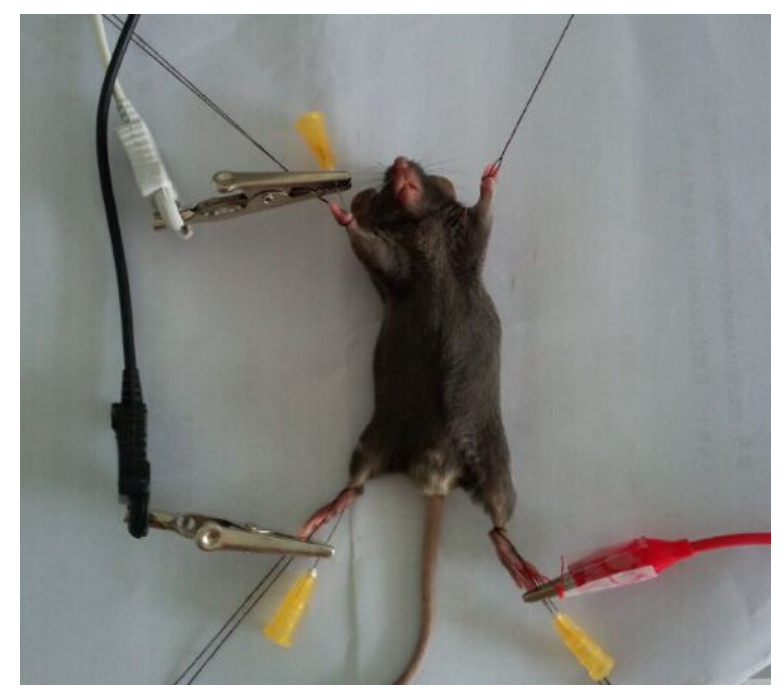

Fig. 3. ECG detection: The experiment use the BIOPAC multi-channel physiological recorder to collect the mouse's electrocardiogram signal, the electrode connection method: VIN+ connects the mouse's left leg, VIN- connects the mouse's right upper limb, GND connects the mouse's right leg.

with gauze were placed into the bottle to absorb the carbon dioxide produced by mice breathing. Stopwatch was used to record the time of hypoxia tolerance test (see Fig. 4).

\subsection{Quantification of IL-1 $\beta$ levels in mouse sera}

Blood samples of mice were collected and centrifuged at $3000 \mathrm{r} / \mathrm{min}$ for $10 \mathrm{~min}$ to obtain the

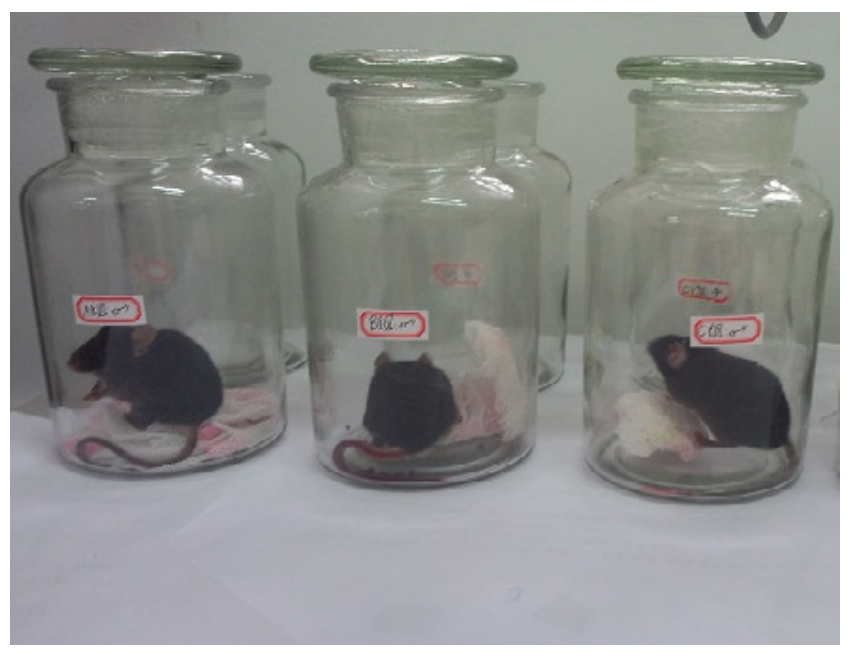

Fig. 4. Normobaric hypoxia tolerance test: All mice were put into a $250 \mathrm{~mL}$ wide mouth bottle filled with $20 \mathrm{~g}$ soda lime (each bottle only 1 mice), and then quickly covered and timing starts until the mice stopped breathing. serum, which were stored at $-40^{\circ} \mathrm{C}$ until use. The level of IL- $1 \beta$ in the serum was determined by enzyme linked immunosorbent assay (ELISAReady-SET-Go, eBioscience) according to the manufacturer's instructions.

\section{Results and Discussions}

The activities of mice were observed carefully during the hypoxia resistant experiments: the respiratory frequency was normal at the beginning of the experiment in each group. Fifteen minutes later, the mice appeared diverse degree of breath shortness. The mice in Control group and Hollow group breath heavier than those in Laser group. In the hypoxia tolerance test, Control group and Hollow group mice became more restless, breathing more and more rapidly, while Laser group mice were only rapidly breathing. After $20 \mathrm{~min}$, Control group and Hollow group mice were with the defecating act, and then got killed. About 3 min later, the mice in Laser group were dead after the appearance of the defecating.

By analyzing records of data, we observed that the range of tolerance time for hypoxia in Control group mice is $1096 \mathrm{~s}-1304 \mathrm{~s}$ and the average of resistance hypoxia time is $1185.67 \mathrm{~s}$; in Hollow group mice, the hypoxia tolerance time range is $1047 \mathrm{~s}-$ $1414 \mathrm{~s}$, and the average resistance to hypoxia time is $1211.5 \mathrm{~s}$; while in the mice of Laser group, the hypoxia tolerance time range is $1096 \mathrm{~s}-1376 \mathrm{~s}$ and the average hypoxia tolerance time is: $1234.83 \mathrm{~s}$ (Fig. 5). It seems that laser acupuncture with optical fiber acupuncture needle intra body may prolong the tolerance to hypoxia in mice. Our preliminary results suggest that laser acupuncture treatment for mice seems favorable to enhance the robustness of the mice body. This conclusion requires reexamination with more precise system such as treating the mice with different wavelength, power, and irradiation time of laser.

The data of ECG detection show that the average heart rates of Control group and Hollow group were 526 and 482 beats/min respectively, while the average heart rate of Laser group was 437 beats/ min (Fig. 6). This is consistent with the observations above Hypoxia, a condition resulting from a deficiency of available oxygen in the body or a region of the body, is responsible for a large number of inflammatory diseases. Inflammation is a defensive 


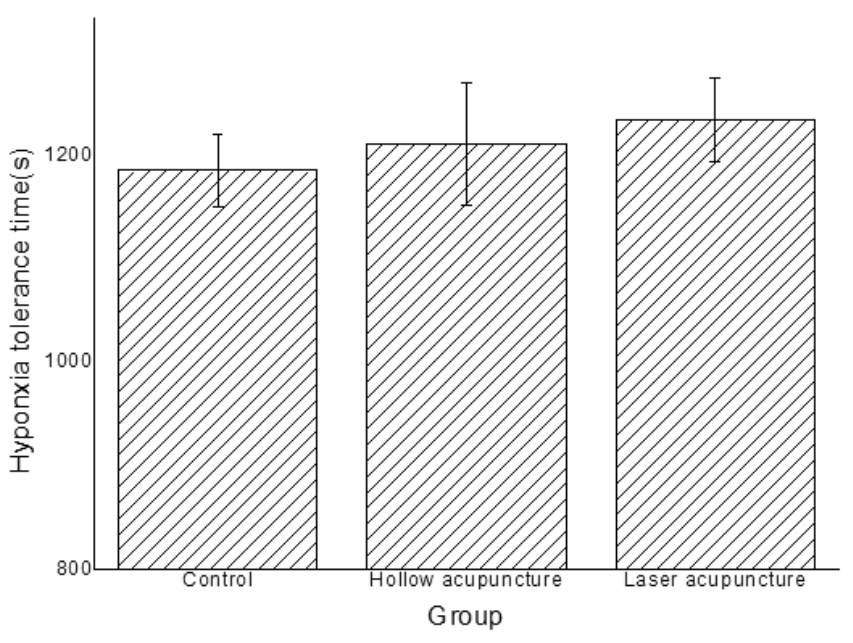

Fig. 5. Through the analysis of experimental data, we found that the mean tolerance to hypoxia in each group was as follows: Control group's average resistance hypoxia time is $1185.67 \mathrm{~s}$; Hollow group's average resistance hypoxia time is $1211.5 \mathrm{~s}$; Laser group's average resistance hypoxia time is $1234.83 \mathrm{~s}$. This shows that laser acupuncture is helpful to prolong the hypoxia tolerance time of mice. The values presented are mean \pm S.E.M. Data were analyzed using ANOVA and multiple comparison. There was no significant difference between Control group and Hollow group $(p=0.714>0.05)$, between Control group and Laser group $(p=0.377>0.05)$, and between Hollow group and Laser group $(p=0.948>0.05)$.

response of body tissues to clear out the harmful stimuli, such as invading pathogens and damaged tissues. However, excessive inflammation may lead to the tissue destruction and death of organism.

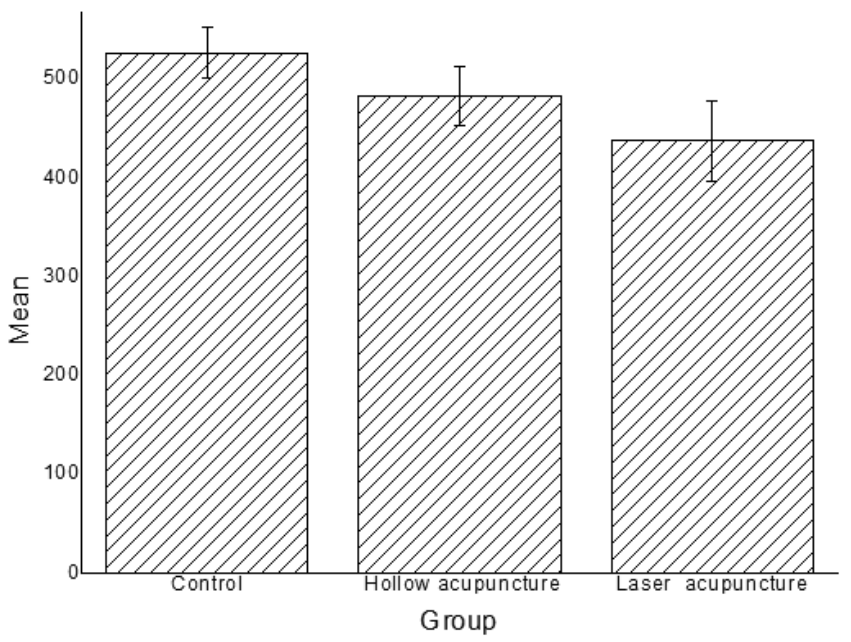

Fig. 6. ECG data analysis. The values presented are mean \pm S.E.M. Data were analyzed using ANOVA and multiple comparison. There was no significant difference between Control group and Hollow group $(p=0.311>0.05)$, between Control group and Laser group $(p=0.093>0.05)$, and between Hollow group and Laser group $(p=0.438>0.05)$.
Among the cytokine families, the members of IL-1 family are mainly associated with inflammation. ${ }^{19}$ Especially, blocking IL-1 $\beta$ has been the standard of therapy for autoinflammatory diseases. ${ }^{20}$

To evaluate whether hypoxia tolerance changes correlates with immune response, we compared the levels of cytokine IL- $1 \beta$, an important mediator of the inflammatory response, in sera of mice after treatment with laser acupuncture, hollow acupuncture, and the control at the time points when the mice were dead. In contrast to the hollow acupuncture or Control group, the level of IL-1 $\beta$ in the laser-acupuncture group was significantly higher indicating that mice treated with laser acupuncture may affect immune response (Fig. 7).

ST36, as foot yangming meridian acupoints, is one of the most important acupoints in clinical acupuncture, and ST36 has been clinically treated for the diseases of the digestive system, the circulatory system and so on. ${ }^{21,22}$ Our results showed that mice in the group that treated with fiber laser acupuncture seemed to prolong the tolerance time to hypoxia and slow down the heart rate. An interesting observation is that the level of IL- $1 \beta$ in the serum of the same group is relatively high. Whether this is the reason accounts for the extended tolerance time to hypoxia is not known. Whether
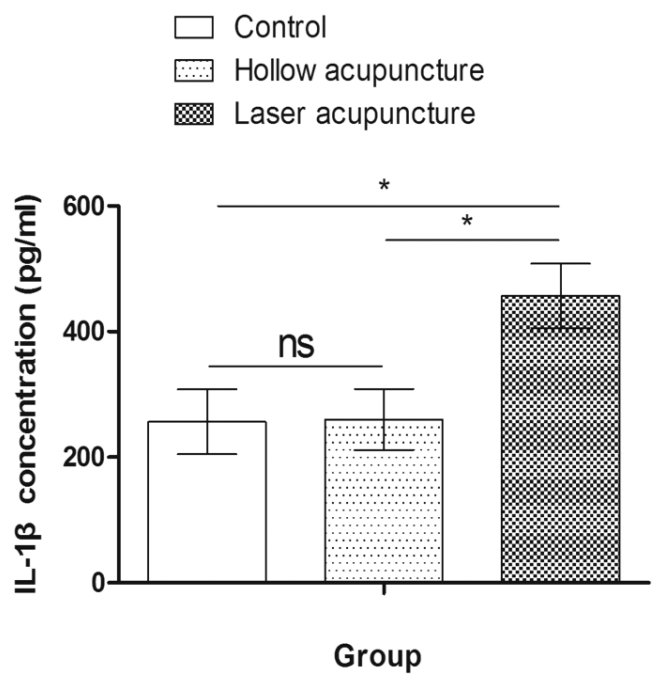

Fig. 7. IL-1 $\beta$ expression in serum. The level of IL- $1 \beta$ in serum were detected. The values presented are mean \pm S.E.M. Data were analyzed using ANOVA and multiple comparison. There was no significant difference between Control group and Hollow group $(p=0.960>0.05)$. There was significant difference between Control group and Laser group $(p=0.033<0.05)$, and between Hollow group and Laser group $(p=0.032<0.05)$.

${ }^{*} P<0.05$; ns: $P>0.05$. 
hypoxia tolerance changes correlates with immune response will be an interesting question for future studies.

\section{Conclusion}

In this paper, we set up an optical fiber-fabricated acupuncture needle device and applied the laser acupuncture treatment on ST36 of mice through intra body experiments. We compared the hypoxia tolerance of mice in Contrast group and Hollow acupuncture group, and found that mice in the group that treated with fiber laser acupuncture seemed to prolong the tolerance time to hypoxia and slow down the heart rate. In addition, we found that mice treated with optical fiber-fabricated acupuncture needle expressed high level of IL- $1 \beta$ in serum. The results of this study are less conclusive with respect to the mechanism underlying the effect of tolerance to hypoxia by laser acupuncture. Many further works such as wavelength, power, treatment frequency and the irradiation time of laser still need to be optimized in order to improve the therapy effectivity.

\section{Acknowledgments}

This work was supported by the Natural Science Foundation of China (NSFC) $(61107076,61177088$, 61475095, 61027015 and 31571430), Shanghai Pujiang Program (14PJ1407700), Health and Family Planning Commission Foundation of Shanghai (201440300), National Program on Key Basic Research Project (973 Program, 2012CB723405, 2014CB541804), the support of the Key Laboratory of Specialty Fiber Optics and Optical Access Networks (SKLSFO2013-02 and SKLSFO2015-01).

\section{References}

1. H. Skjeie, T. Skonnord, A. Fetveit, M. Brekke, "Acupuncture for infantile colic: A blinding-validated, randomized controlled multicentre trial in general practice," Scand. J. Prim. Health Care 31, 190-196 (2013).

2. G. X. Shi, X. M. Yang, C. Z. Liu, L. P. Wang, "Factors contributing to therapeutic effects evaluated in acupuncture clinical trials," Trials 13, 42 (2012).

3. D. J. Ramsay, M. A. Bowman, "Acupuncture," Jama-J. Am. Med. Assoc. 280, 1518-1524 (1998).
4. C. Sutalangka, J. Wattanathorn, S. Muchimapura, W. Thukham-Mee, P. Wannanon, T. Tong-un, "Laser acupuncture improves memory impairment in an animal model of Alzheimer's disease," $J$. Acupunct. Meridian Stud. 6, 247-251 (2013).

5. I. Quah-Smith, W. Wen, X. Chen, M. A. Williams, P. S. Sachdev, "The brain effects of laser acupuncture in depressed individuals: An fMRI investigation," Med. Acupunct. 24, 161-171 (2012).

6. I. Quah-Smith, M. A. Williams, T. Lundeberg, C. Suo, P. Sachdev, "Differential brain effects of laser and needle acupuncture at LR8 using functional MRI," Acupunct. Med. 31, 282-289 (2013).

7. I. Quah-Smith, P. S. Sachdev, W. Wen, X. Chen, M. A. Williams, R. Musil, "The brain effects of laser acupuncture in healthy individuals: an FMRI investigation," Dtsch. Z. Akupunkt. 54, 22-23 (2011).

8. E. Sari, T. Sari, "The role of acupuncture in the treatment of orthodontic patients with a gagging reflex: a pilot study," Br. Dent. J. 208, E19 (2010).

9. J. C. Turp, "Limited evidence that acupuncture is effective for treating temporomandibular disorders," Evid. Based Dent. 12, 89 (2011).

10. S. H. Cho, J. S. Lee, L. Thabane, J. Lee, "Acupuncture for obesity: A systematic review and meta-analysis," Int. J. Obes. 33, 183-196 (2009).

11. I. Quah-Smith, P. S Sachdev, W. Wen, X. H. Chen, A. Mark A. Williams, "The brain effects of laser acupuncture in healthy individuals: An fMRI investigation," PLoS One, 5(9): e12619 (2010).

12. P. Whittaker, "Laser acupuncture: Past, present, and future," Lasers Med. Sci. 19, 69-80 (2004).

13. G. Litscher, X. Y. Gao, L. Wang, B. Zhu, High-Tech Acupuncture and Integrative Laser Medicine 2014. Evid Based Compl. Alt. Med. 2015, 878620 (2015).

14. W. He, G. Litscher, X. H. Jing, H. Shi, X. Y. Wang, I. Gaischek, Y. S. Su, D. Litscher, Z. K. Yang, J. J. Xin, L. Hu, "Effectiveness of interstitial laser acupuncture depends upon dosage: Experimental results from electrocardiographic and electrocorticographic recordings," Evid. Based Compl. Alt. Med. 2013, 934783 (2013).

15. G. Litscher, D. Rachbauer, S. Ropele, L. Wang, D. Schikora, F. Fazekas, F. Ebner, "Acupuncture using laser needles modulates brain function: First evidence from functional transcranial doppler sonography and functional magnetic resonance imaging," Lasers Med. Sci. 19(1), 6-11 (2004).

16. A. L. Marinela, G. Litscher, M. Weber, C. Ailioaie, D. Litscher, D. A. Chiran, "Innovations and challenges by applying sublingual laser blood irradiation in juvenile idiopathic arthritis," Int. J. Photoenergy 2014, 1-8 (2014). 
17. D. A. Chiran, M. Weber, L. M. Ailioaie, E. Moraru, C. Ailioaie, D. Litscher, G. Litscher, "Intravenous laser blood irradiation and tocilizumab in a patient with juvenile arthritis," Case Rep. Med. 2014, 923496 (2014).

18. W. He, D. Wedig, L. Wang, I. Gaischek, G. Litscher, "Violet laser acupuncture - Part 5: An investigation of different stimulation frequencies on heart rate and variability," J. Acupunct. Meridian Stud. 5(6), 290-294 (2012).

19. C. A. Dinarello, "Interleukin-1 in the pathogenesis and treatment of inflammatory diseases," Blood $\mathbf{1 1 7}$ (14), 3720-3732 (2011).
20. S. L. Masters, A. Simon, I. Aksentijevich, D. L. Kastner,. "Horror autoinflammaticus: the molecular pathophysiology of autoinflammatory disease," Ann. Rev. Immunol. 27, 621-668 (2009).

21. Z. R. Sun, "Effect of inserting zusanli (ST36) on cardiac function of rats," Information on TCM, 5(1), 40 (1988).

22. H. X. Zhang, C. F. Li, "Electro-needling at 'Zusanli' in rabbits its effect on blood pressure and heart rate and the possible mechanism," Acupunct. Res. 10(4), 304-308 (1985). 